\title{
Changes in lower urinary tract symptoms after prostate brachytherapy
}

\author{
Bruce L. Jacobs, MD', Ryan P. Smith, MD², Sushil Beriwal, MD², Ronald M. Benoit, MD \\ 'Department of Urology, 2Department of Radiation Oncology, University of Pittsburgh School of Medicine, Pittsburgh, Pennsylvania, USA
}

\begin{abstract}
Purpose: To further define the bothersome lower urinary tract symptoms that occur after prostate brachytherapy $\mathrm{PB}$ ) by evaluating patient's responses to the individual questions of the urinary portion of the Expanded Prostate Cancer Index Composite (EPIC) survey and the AUA symptoms score in men undergoing PB.

Material and methods: A longitudinal, prospective study of 170 patients who have undergone PB at a single institution was performed. All patients were asked to complete the EPIC survey pre-operatively and at 2 weeks, 4 weeks, 3 months, and 6 months post-operatively. Starting with the $75^{\text {th }}$ patient in the cohort, patients were also asked to complete the AUA symptom score.

Results: The pattern of changes for each question is similar for both the EPIC survey and the AUA symptom score, with a marked worsening of symptoms at 2 and 4 weeks and an improvement to baseline by 3 to 6 months. Hematuria questions had the quickest and dysuria questions had the longest return to baseline. The dysuria questions had the greatest change and the incontinence questions had the smallest change in magnitude. Obstructive symptoms had a greater magnitude of change when compared to irritative symptoms, but the irritative symptoms took longer to return to baseline.

Conclusions: The present study adds to the fund of knowledge regarding the bothersome lower urinary tract symptoms which occur after PB by analyzing the individual questions of both the urinary portion of the EPIC survey and the AUA symptom score.

Key words: brachytherapy, cesium-131, EPIC, quality of life.

\section{Purpose}

Prostate cancer remains the most common solid tumor diagnosed in men in the United States, as an estimated 217730 cases were diagnosed in 2010 with an estimated 32050 deaths [1]. Prostate brachytherapy (PB) is one of several accepted treatment options and has been shown to be an effective treatment for clinically localized disease [2]. Several centers have published outcomes with long-term follow-up, demonstrating excellent cancer control with prostate-specific antigen (PSA) relapse-free survival equivalent to radical prostatectomy and external beam radiotherapy [3-5]. Given the lack of clear superiority in cancer control among the different treatment options and the slow growing nature of prostate cancer, consideration of healthrelated quality of life after treatment has become a very important factor as men decide which treatment option to pursue [6]. Patients who choose PB are attracted to the minimally invasive nature of the procedure, the quick return to full activity, and the low risk of long-term urinary incontinence. However, bothersome lower urinary tract symptoms are quite common after PB. These symptoms can persist for greater than 12 months after the procedure and can significantly affect quality of life [7-9]. Most men will experience at least some change in their voiding pattern after prostate brachytherapy [10-12]. These symptoms can include frequency, urgency, urge incontinence, nocturia, dysuria, a weakened force of the urinary stream, hesitancy, and intermittency. Urinary morbidity after PB was initially reported using the Radiation Therapy Oncology Group (RTOG) criteria [13, 14]. To improve understanding of the lower urinary tract symptoms which occur after $\mathrm{PB}$, centers began reporting results of patient recorded symptom surveys such as the AUA symptom score and the EPIC survey $[15,16]$. These studies generate a total score based on patient responses to the individual questions of the survey. These scores can be used to demonstrate the magnitude and duration of the changes in symptoms that occur after prostate brachytherapy, but do not provide an understanding of the specific symptoms that can occur.

The present study attempts to further define the bothersome lower urinary tract symptoms that occur after prostate brachytherapy by reporting the results of individual questions to both the AUA symptom score and the urinary 
portion of the EPIC survey. By reporting the results of the individual questions of this survey, we hope to provide to both physicians performing prostate brachytherapy and patients considering this procedure more information as to how specific lower urinary tract symptoms are affected by prostate brachytherapy.

\section{Material and methods}

All men in our prostate brachytherapy program were asked to complete the urinary and bowel portion of the EPIC survey prior to their procedure. Patients were asked to return 2 weeks, 4 weeks, 3 months, and 6 months after their procedure. At the time of these follow up visits, patients were requested to complete again the EPIC survey. Beginning with the $75^{\text {th }}$ patient in this cohort, patients were also asked to complete the AUA symptom score in addition to the EPIC survey. The 50-item EPIC survey, which is based on modifications of the University of California-Los Angeles Prostate Cancer Index, has shown satisfactory survey characteristics in validation analyses, has test-retest reliability, and internal consistency for urinary, bowel, sexual, and hormonal domains. The EPIC survey specifically addresses both urinary and bowel bother and function components [15]. Each domain is scored from 0 to 100, with higher scores representing less bothersome symptoms. The urinary portion of the EPIC survey consists of two parts. The first part contains five questions regarding voiding symptoms. The second part contains seven questions which inquire about the degree of bother caused by these symptoms. These questions are listed in Appendix A.

The AUA symptom score consists of seven questions regarding frequency, nocturia, the force of the urinary stream, hesitancy, intermittency, incomplete emptying, and urgency [16]. This instrument has been shown to be internally consistent with excellent test-retest reliability. The scores are highly correlated with a patient's global rating of the magnitude of their urinary symptoms. The score has also been demonstrated to be sensitive to change. The AUA symptom score has proven to be valuable in both the clinical and the research setting. Additionally, the AUA symptom score can be further stratified into an irritative (nocturia, frequency, urgency) and obstructive (force of stream, intermittency, hesitancy, incomplete emptying) component. The questions of the AUA symptom score are listed in Appendix B.

Our program utilizes the Cesium-131 $\left({ }^{131} \mathrm{Cs}\right)$ isotope (IsoRay Medical, Richland, $\mathrm{WA}^{\circledR}$ ) [17]. The isotope was implanted using real-time planning. Seeds were placed by afterloading with a Mick applicator ${ }^{\circledR}$. Prostate volume was measured pre-operatively (via either computed tomography or ultrasound) in order to estimate the activity required. At the time of the implant, the prostate volume was measured using a step section technique at $5 \mathrm{~mm}$ intervals from the base of the prostate to the apex. Peripheral needles were placed first, approximately $8-10 \mathrm{~mm}$ apart and just under the capsule of the prostate in the largest transverse image, and 2 to 8 central needles (depending on prostate volume and geometry) were then placed to ensure adequate dosing of the central portion of the gland. Posterior needles were kept approximately $5 \mathrm{~mm}$ from the posterior prostate cap- sule. After all needles were placed, post-needle prostate volume was obtained by again contouring the prostate in transverse sections in $5 \mathrm{~mm}$ intervals from base to apex. Realtime intra-operative dosimetry (VariSeed 7.1, Varian Medical Systems, Palo Alto, CA, USA ${ }^{\circledR}$ ) was used to determine the location of seed placement based on the postneedle prostate volume and geometry on ultrasound. A modified peripheral loading technique was utilized. Seeds were placed with a Mick applicator. Goals of intraoperative live planning were: D90 > 110\%, V100 > 90\%, $\mathrm{V} 150<50 \%$, and V200 < 25\%. Planned dose was 115 Gy for men undergoing prostate brachytherapy as monotherapy, and $85 \mathrm{~Gy}$ for men undergoing prostate brachytherapy as part of combined radiotherapy with external beam radiotherapy. Computed tomography (CT) based dosimetry was obtained on day 0 . Men undergoing combined radiotherapy underwent prostate brachytherapy approximately four weeks after their EBRT was completed. Steroids were not used peri-operatively and men were not routinely started on alpha blockade prior to their procedure.

The twelve questions of the EPIC urinary survey and the seven questions of the AUA symptom score were analyzed individually. The mean score for each question was reported at each time point. Additionally, obstructive and irritative components of the AUA symptom score were constructed and analyzed. Comparisons between time points for individual questions were compared using the Student's $t$ test with a $p$-value of $<0.05$, considered statistically significant.

\section{Results}

One hundred seventy men underwent prostate brachytherapy during the time period of this study. All men in the study completed a pre-operative EPIC survey, while 150 men (88.2\%), 99 men (58.2\%), 156 men (91.8\%), and 115 men (67.6\%), completed surveys at 2 weeks, 4 weeks, 3 months, and 6 months, respectively. We began asking men to complete the AUA symptom score in addition to the EPIC survey beginning with the $75^{\text {th }}$ patient in this cohort, and therefore have serial AUA symptom scores in 95 of the men in this study. Of these men, 83 men $(87.4 \%), 39$ men (41.1\%), 90 men $(94.7 \%)$, and 67 men $(70.5 \%)$, completed AUA symptom scores at 2 weeks, 4 weeks, 3 months, and 6 months, respectively. The decision to add the AUA symptom score to our patient evaluation was based on our desire to compare our outcomes to other studies in the brachytherapy literature which use the AUA symptom score to monitor their patients symptoms. Clinical information regarding these patients appears in Table 1 . Mean age was 66.0 years and mean pre-treatment PSA was $6.7 \mathrm{ng} / \mathrm{ml}$. One hundred and nineteen men in the study underwent PB as monotherapy, while 51 men underwent PB as part of combination therapy with external beam radiotherapy. Androgen deprivation therapy was administered to 27 patients. The mean pre-operative EPIC urinary summary score was 86.6, and the mean AUA symptom score was 6.6.

Post-implant dosimetry and prostate volume presents Table 2. Mean D90 was $106.8 \%$, and mean V100 was $92.5 \%$. Mean maximum urethral dose was $167.2 \mathrm{~Gy}$, and mean urethral D100 and mean urethral D150 were $1.0 \mathrm{cc}$ and $0.04 \mathrm{cc}$, respectively. Mean prostate volume was $41.8 \mathrm{cc}$ with 
Table 1. Clinical information on the 170 patients included in the study

\begin{tabular}{ll}
\hline PB as monotherapy & $110(65 \%)$ \\
\hline XRT + PB & $33(19 \%)$ \\
\hline XRT + PB + ADT & $18(11 \%)$ \\
\hline PB + ADT & $9(5 \%)$ \\
\hline Mean age (years) & $66.0($ range 50-83) \\
\hline Mean PSA (ng/ml) & 6.7 (range 1.1-78) \\
\hline Median Gleason Score & 7 (range 6-9) \\
\hline Low risk & $75(44 \%)$ \\
\hline Intermediate risk & $74(44 \%)$ \\
\hline High risk & $21(12 \%)$ \\
\hline Clinical stage T1C & $136(80 \%)$ \\
\hline Clinical stage T2A & $19(11 \%)$ \\
\hline Clinical stage T2B & $11(6 \%)$ \\
\hline Clinical stage T2C & $4(2 \%)$
\end{tabular}

$P B$ - prostate brachytherapy, XRT-external beam radiotherapy, $A D T$ - androgen deprivation therapy

Low risk $=P S A<10 \mathrm{ng} / \mathrm{ml}$ and Gleason score $\leq 6$; intermediate risk = PSA $\geq 10 \mathrm{ng} / \mathrm{ml}$ or Gleason score 7; high risk $=P S A \geq 10 \mathrm{ng} / \mathrm{ml}$ and Gleason score $\geq 10 \mathrm{ng} / \mathrm{ml}$ or PSA $\geq 20 \mathrm{ng} / \mathrm{ml}$ or Gleason score $\geq 8$ [20]

Clinical staging is based on the American Joint Commission on Cancer Staging Tstage [21]

Table 3. The mean value of the EPIC survey score for the entire cohort for each question at each time point studied. The questions are listed in Appendix A

\begin{tabular}{|c|c|c|c|c|c|}
\hline EPIC & Pre-op & 2 weeks & 4 weeks & 3 months & 6 months \\
\hline Summary & 86.8 & 58.7 & 62.0 & 78.6 & $82.9^{*}$ \\
\hline 1 & 86.5 & 69.5 & 45.2 & $74.0^{*}$ & $85.5^{\star}$ \\
\hline 2 & 99.6 & 72.5 & 90.2 & $98.6^{*}$ & $99.1^{*}$ \\
\hline 3 & 94.6 & 31.8 & 34.6 & 81.1 & 80.9 \\
\hline 4 & 88.7 & 74.0 & 77.0 & 80.2 & $85.9^{*}$ \\
\hline 5 & 98.5 & 90.6 & 92.9 & 91.9 & $97.2^{*}$ \\
\hline 6 & 89.4 & 74.8 & 78.8 & 82.3 & $85.8^{\star}$ \\
\hline 7 & 93.9 & 46.5 & 48.5 & 82.9 & 85.3 \\
\hline 8 & 99.7 & 81.2 & 89.7 & $99.0^{*}$ & $97.8^{*}$ \\
\hline 9 & 77.7 & 42.7 & 41.7 & 67.7 & $76.6^{*}$ \\
\hline 10 & 65.7 & 36.5 & 36.9 & 56.1 & $62.9^{*}$ \\
\hline 11 & 68.4 & 39.2 & 38.4 & 61.4 & $68.3^{*}$ \\
\hline 12 & 76.6 & 40.7 & 38.6 & 63.5 & $71.8^{\star}$ \\
\hline
\end{tabular}

*Indicates value is not statistically significant different from baseline $(p<0.05)$

a range of $14.9 \mathrm{cc}$ to $89.1 \mathrm{cc}$. At the time of diagnosis of prostate cancer, $33(19.4 \%)$ patients were maintained on alpha-blockade and of the remaining 137 patients, $103(75.2 \%)$ men were placed on alpha-blockade after their procedure. The mean value for each question at each time point for the urinary portion of the EPIC survey appears in Table 3. The greatest decrease from baseline occurred in a dysuria question (question 3 ). The mean preoperative value for this question decreased by 62.8 two
Table 2. Post-implant dosimetry and prostate volume

\begin{tabular}{lccc} 
& Mean & Median & $\begin{array}{l}\text { Standard } \\
\text { deviation }\end{array}$ \\
\hline Prostate D90 & $106.8 \%$ & $104.6 \%$ & 10.0 \\
\hline Prostate V100 & $92.5 \%$ & $93.2 \%$ & 4.5 \\
\hline Prostate V150 & $45.4 \%$ & $44.5 \%$ & 12.9 \\
\hline Prostate V200 & $17.2 \%$ & $15.5 \%$ & 7.2 \\
\hline Maximum urethral dose & $167.2 \mathrm{~Gy}$ & $162.6 \mathrm{~Gy}$ & 40.5 \\
\hline Urethral V100 & $1.0 \mathrm{cc}$ & $0.98 \mathrm{cc}$ & 0.35 \\
\hline Urethral V150 & $0.04 \mathrm{cc}$ & $0 \mathrm{cc}$ & 0.13 \\
\hline Prostate volume & $41.8 \mathrm{cc}$ & $39.8 \mathrm{cc}$ & 15.4
\end{tabular}

Prostate D90 is the radiation dose delivered to $90 \%$ of the prostate

Prostate V100 is the volume of the prostate receiving 100\% of the prescribed dose Prostate V150 is the volume of the prostate receiving $150 \%$ of the prescribed dose Prostate V200 is the volume of the prostate receiving 200\% of the prescribed dose Urethral V100 is the volume of the urethra receiving 100\% of the prescribed dose Urethral V150 is the volume of the urethra receiving $150 \%$ of the prescribed dose 
Table 4. The mean value of the AUA symptom score for the entire cohort for each question at each time point studied. The questions are listed in Appendix B

\begin{tabular}{|c|c|c|c|c|c|}
\hline AUA & Pre-op & 2 weeks & 4 weeks & 3 months & 6 months \\
\hline Total Score & 6.6 & 17.5 & 19.8 & 10.3 & $8.0^{*}$ \\
\hline 1 & 0.7 & 2.4 & 2.5 & 1.2 & $0.9^{*}$ \\
\hline 2 & 1.4 & 3.0 & 3.3 & 1.8 & $1.5^{*}$ \\
\hline 3 & 0.8 & 2.3 & 2.7 & 1.3 & $0.9^{\star}$ \\
\hline 4 & 0.8 & 2.1 & 2.8 & 1.8 & 1.3 \\
\hline 5 & 0.9 & 3.0 & 3.0 & 1.7 & $1.1^{\star}$ \\
\hline 6 & 0.4 & 1.9 & 2.3 & 0.63 & $0.5^{\star}$ \\
\hline 7 & 1.6 & 2.9 & 3.2 & 2.0 & $1.8^{*}$ \\
\hline Irritative & 3.8 & 8.0 & 9.3 & 5.5 & 4.6 \\
\hline Obstructive & 2.8 & 9.5 & 10.5 & 4.8 & $3.3^{*}$ \\
\hline
\end{tabular}

*Indicates value is not statistically significant different from baseline $(p<0.05)$

cedure. By six months after the procedure, mean values for the incontinence questions differed from baseline by 1.0, 2.8, 1.3 , and 3.6, respectively, and the differences were not statistically significant. The difference in mean value for question 1 was not statistically significant from baseline by 3 months after the procedure. The quickest return to baseline occurred in the hematuria questions. The change in mean value for the two hematuria questions from baseline to 2 weeks after the procedure was 27.1 and 18.5. The scores for these questions were not significantly different from baseline by 3 months after the procedure. The question which deals with obstructive symptoms had a mean value of 77.7 at baseline, and decreased to 42.7 at 2 weeks, decreased further to 41.7 at 4 weeks, increased to 67.7 at 3 months, and to 76.6 at 6 months. Questions 10 (nocturia) and 11 (frequency) had changes in magnitude and duration similar to the obstructive question.

The mean value for each question in the AUA symptom score at each time point appears in Table 4 . The questions dealing with obstructive symptoms all had a greater change from baseline at 2 weeks than the questions dealing with irritative symptoms. However, the only value that did not return to statistical baseline by 6 months was question 4 (urgency). By combining the four obstructive questions (straining, incomplete emptying, stream, intermittency), an obstructive component was created and similarly, an irritative component was created by combining the three irritative questions (urgency, frequency, nocturia). The mean value of the obstructive component had a greater change at 2 weeks, but the irritative component had a slower return to baseline. The mean value for the irritative component had not yet returned to statistical baseline by 6 months after the procedure.

\section{Discussion}

Patients who undergo prostate brachytherapy are drawn to the minimally invasive nature of the procedure, the quick return to full activity, and the low risk of urinary incontinence. However, bothersome lower urinary tract symptoms occur commonly after the procedure. Initially, urinary morbidity after prostate brachytherapy was reported using RTOG criteria $[13,14]$. In hopes of providing a better description of the lower urinary tract symptoms after prostate brachytherapy, studies began utilizing patient reported symptom surveys such as the AUA symptom score and the EPIC survey. These instruments generate a total score based on responses to the individual questions of the survey. The present study analyzes responses to the individual questions of these surveys in hopes of furthering our understanding of the changes in lower urinary tract symptoms that occur after prostate brachytherapy. The pattern of changes is similar for most of the EPIC questions. The general trend is a marked decrease at 2 weeks and 4 weeks after the procedure, followed by an improvement at 3 months and a return to statistical baseline by 6 months. The only questions of the urinary portion of the EPIC survey which did not return to statistical baseline by 6 months were questions 3 and 7 , both of which deal with dysuria. The two questions dealing with hematuria returned to statistical baseline by 3 months after the procedure. The other question on the urinary portion of the EPIC survey which returned to statistical baseline by 3 months was question 1 (How often have you leaked urine?). However, the magnitude of these changes differs. The greatest change at two weeks after the procedure occurs in the dysuria questions (a decrease of $50.5 \%$ to $66.4 \%$ from baseline). The smallest change two weeks after the procedure occurs in the incontinence questions (a decrease of $8.0 \%$ to $19.7 \%$ from baseline). Questions dealing with urinary frequency, nocturia, and obstructive symptoms demonstrate a decrease from baseline values of $42.7 \%$ to $45.0 \%$ at 2 weeks and $43.9 \%$ to $46.3 \%$ at 4 weeks. These values then increased, and were only $10.2 \%$ to $13.7 \%$ decreased from baseline at 3 months, and were $0.1 \%$ to $4.3 \%$ decreased from baseline at 6 months.

The AUA symptom score does not address dysuria, incontinence or hematuria, but rather focuses on irritative (3 questions) and obstructive symptoms (4 questions). Patients reported a greater magnitude of change in the obstructive questions of the AUA symptom score. Of the sev- 
en questions, all four obstructive questions had a greater percentage change from baseline at 2 weeks than the three irritative questions. The greatest change at 2 weeks $(78.9 \%$ from baseline) occurred in question 6 (straining to void), followed by question 1 (incomplete emptying) at $70.8 \%$, question 5 (weak stream) at $70.0 \%$, and question 3 (intermittency) at $65.2 \%$. The irritative questions had a lower percentage change at 2 weeks after the procedure when compared to baseline as question 4 (urgency) decreased $61.9 \%$, question 2 (frequency) decreased $53.3 \%$, and question 7 (nocturia) decreased $44.8 \%$.

Although the obstructive questions had a greater change from baseline at 2 weeks, the irritative questions generally took longer to return to baseline. Despite the urgency question having a smaller decrease initially than all of the obstructive questions, the mean value of this question increased at a slower rate over time than the other 6 questions. The urgency question was the only AUA symptom score question which did not return to statistical baseline by 6 months after the procedure. The two questions that had the greatest absolute difference from baseline at 6 months addressed the irritative symptoms (the urgency question ( 0.47 from baseline) and the nocturia question ( 0.23 from baseline). All other questions had a difference from baseline at 6 months of 0.16 or less.

Patients in the present study underwent prostate brachytherapy with $\mathrm{Cs}^{131}$. Therefore, the results of this study can not be readily transferred to patients who are undergoing prostate brachytherapy with Iodine 125 or Palladium 103. The shorter half-life of Palladium (17 days) has been proposed to lead to a shorter duration, but greater magnitude of the lower urinary tract symptoms after prostate brachytherapy when compared to patients undergoing prostate brachytherapy with Iodine (half-life of 60 days) [18], although other studies have not demonstrated such differences between these two isotopes [19]. While the magnitude of the changes in lower urinary tract symptoms and duration of these changes may differ between men undergoing prostate brachytherapy with Cesium, Iodine, and Palladium; the pattern of a sharp initial worsening of these symptoms followed by a gradual improvement to baseline is likely to be similar. No evidence exists to suspect that the specific symptoms (e.g. obstructive, irritative, incontinence, dysuria) will differ among the available isotopes.

\section{Conclusions}

Knowledge of treatment sequelae of the available therapeutic strategies for prostate cancer is essential for counseling patients as they consider their treatment options. The present study adds to the fund of knowledge regarding the bothersome lower urinary tract symptoms which occur after prostate brachytherapy by analyzing the individual questions of both the urinary portion of the EPIC survey and the AUA symptom score. The general pattern is a marked worsening of scores for these questions at 2 weeks and 4 weeks after the procedure, with an improvement at 3 months, and a further improvement to statistical baseline by 6 months after the procedure. The magnitude of these changes is greatest for dysuria and smallest for incontinence, with irritative and obstructive symptoms assuming a mid- dle ground between these two symptoms. When obstructive and irritative symptoms are compared, obstructive symptoms have a greater decrease in magnitude from baseline, while irritative voiding symptoms take longer to return to baseline.

\section{Disclosure}

Authors reported no actual or potential conflicts of interest. Role of the funding source: not applicable.

\section{References}

1. Jemal A, Siegel R, Xu J et al. Cancer statistics, 2010. CA Cancer J Clin 2010; 60: 277-300.

2. Cesaretti JA, Stone NN, Skouteris VM et al. Brachytherapy for the treatment of prostate cancer. Cancer J 2007; 13: 302-312.

3. Joseph J, Al-Qaisieh B, Ash D et al. Prostate-specific antigen relapse-free survival in patients with localized prostate cancer treated by brachytherapy. BJU Int 2004; 94: 1235-1238.

4. Grimm PD, Blasko JC, Sylvester JE et al. 10-year biochemical (prostate-specific antigen) control of prostate cancer with (125) I brachytherapy. Int J Radiat Oncol Biol Phys 2001; 51: 31-40.

5. Zelefsky MJ, Hollister T, Raben A et al. Five-year biochemical outcome and toxicity with transperineal CT-planned permanent I-125 prostate implantation for patients with localized prostate cancer. Int J Radiat Oncol Biol Phys 2000; 47: 1261-1266.

6. Penson DF, Litwin MS, Aaronson NK. Health related quality of life in men with prostate cancer. J Urol 2003; 169: 1653-1661.

7. Van Gellekom MP, Moerland MA, Van Vulpen M et al. Quality of life of patients after permanent prostate brachytherapy in relation to dosimetry. Int J Radiat Oncol Biol Phys 2005; 63: 772-780.

8. Ash D, Bottomley D, Al-Qaisieh B et al. A prospective analysis of long-term quality of life after permanent I-125 brachytherapy for localized prostate cancer. Radiother Oncol 2007; 84: 135-139.

9. Talcott JA, Manol J, Clark JA et al. Time course and predictors of symptoms after primary prostate cancer therapy. J Clin Oncol 2003; 21: 3979-3986.

10. Anderson JF, Swanson DA, Levy LB et al. Urinary side effects and complications after permanent prostate brachytherapy: the MD Anderson Cancer Center experience. Urology 2009; 74 : 601-605.

11. Aaltomaa SH, Kataja VV, Lahtinen T et al. Eight years experience of local prostate cancer treatment with permanent I125 seed brachytherapy - morbidity and outcome results. Radiother Oncol 2009; 91: 213-216.

12. Vordermark D, Noe M, Markert K et al. Prospective evaluation of quality of life with I125: importance of baseline symptoms and of prostate-V150. Radiother Oncol 2009; 91: 217-224.

13. Gellum DY, Potters L, Ashley R et al. Urinary morbidity following ultrasound-guided transperineal prostate seed implantation. Int J Radiat Oncol Biol Phys 1999; 45: 59-67.

14. Brown D, Colonias A, Miller R et al. Urinary morbidity with a modified peripheral loading technique of transperineal (125) i prostate implantation. Int J Radiat Oncol Biol Phys 2000; 47: 353-360.

15. Wei JT, Dunn RL, Litwin MS et al. Development and validation of the expanded prostate cancer index composite (EPIC) for comprehensive assessment of health-related quality of life in men with prostate cancer. Urology 2000; 56: 899-905.

16. Barry MJ, Fowler FJ Jr, O'Leary M et al. The American Urological Association symptom index for benign prostatic hyperplasia. The Measurement Committee of the American Urological Association. J Urol 1992; 148: 1549-1557. 
17. IsoRay Medical, Inc, CS- $1{ }^{131}$ Cs product information sheet and company web site. www.isoray.com. Accessed June 8, 2011.

18. Wallner K, Merrick G, True L et al. I-125 versus Pd-103 for lowrisk prostate cancer: morbidity outcomes from a prospective randomized multicenter trial. Cancer J 2005; 11: 385-389.

19. Niehaus A, Merrick GS, Butler WM et al. The influence of isotope and prostate volume on urinary morbidity after prostate brachytherapy. Int J Radiat Oncol Biol Phys 2006; 64: 136-143.

20. D'Amico AV, Whittington R, Malkowicz SB et al. Biochemical outcome after radical prostatectomy, external beam radiation therapy, or interstitial radiation therapy for clinically localized prostate cancer. JAMA 1998; 280: 969-974.

21. Greene FL, Page DL, Fleming ID et al. In: AJCC Cancer Staging Manual, 6th ed. Springer, New York 2002; 309-316. 